\title{
HYPONORMAL POWERS OF COMPOSITION OPERATORS
}

\author{
PHILLIP DIBRELL AND JAMES T. CAMPBELL
}

(Communicated by John B. Conway)

\begin{abstract}
Let $T_{i}, i=1,2$, be measurable transformations which define bounded composition operators $C_{T_{i}}$ on $L^{2}$ of a $\sigma$-finite measure space. Denote their respective Radon-Nikodym derivatives by $h_{i}, i=1,2$. The main result of this paper is that if $h_{i} \circ T_{i} \leq h_{j}, i, j=1,2$, then for each of the positive integers $m, n, p$ the operator $\left[C_{T_{1}}^{m} C_{T_{2}}^{n}\right]^{p}$ is hyponormal. As a consequence, we see that the sufficient condition established by Harrington and Whitley for hyponormality of a composition operator is actually sufficient for all powers to be hyponormal.
\end{abstract}

I. Preliminaries. Let $(X, \Sigma, m)$ be a $\sigma$-finite measure space, and let $T$ be a measurable transformation from $X$ into itself. Set $L^{2}=L^{2}(X, \Sigma, m)$. The equation

$$
C_{T} f=f \circ T, \quad f \in L^{2}
$$

defines a composition transformation from $L^{2}$ to the space of $\mathbf{C}$-valued functions on $X . C_{T}$ is a bounded linear operator on $L^{2}$ precisely when (i) $m \circ T^{-1}$ is absolutely continuous with respect to $m$, and (ii) $h=d m \circ T^{-1} / d m$ is in $L^{\infty}(X, \Sigma, m)=L^{\infty}$. Denote by $R\left(C_{T}\right)$ the range of $C_{T}$, by $C_{T}^{*}$ the adjoint of $C_{T}$, and define $T^{-1}(\Sigma)$ as the relative completion of the $\sigma$-algebra $\left\{T^{-1}(A): A \in \Sigma\right\}$. The following lemma, due to Harrington and Whitley [2, p. 126], is well known and useful.

1.1 Lemma. Let $P$ denote the projection of $L^{2}$ onto $\overline{R\left(C_{T}\right)}$.

(a)

$$
C_{T}^{*} C_{T} f=h f \quad \text { and } \quad C_{T} C_{T}^{*} f=(h \circ T) P f, \quad \text { all } f \in L^{2} .
$$

(b)

$$
\overline{R\left(C_{T}\right)}=\left\{f \in L^{2}: f \text { is } T^{-1}(\Sigma) \text {-measurable }\right\} .
$$

(c) If $f$ is $T^{-1}(\Sigma)$-measurable, and $g$ and $f g$ belong to $L^{2}$, then

$$
P(f g)=f P(g) .
$$

( $f$ need not be in $L^{2}$.)

Finally we recall that a bounded linear operator $A$ on Hilbert space $\mathcal{H}$ is hyponormal if $A^{*} A-A A^{*} \geq 0$ (or equivalently, if $\|A f\| \geq\left\|A^{*} f\right\|$, for each $f \in \mathcal{H}^{\text {.) }}$ Now let $T_{1}$ and $T_{2}$ be measurable transformations of $X$ with R-N derivatives $h_{1}$ and $h_{2}$, respectively, with (i) and (ii) above satisfied by both pairs. It follows that the transformation $T_{3}: X \rightarrow X$ given by $T_{1} \circ T_{2}$ will be measurable, and the R-N

Received by the editors July 1, 1986 and, in revised form, September 22, 1986 and January 26, 1987.

1980 Mathematics Subject Classification (1985 Revision). Primary 47B20; Secondary $47 \mathrm{~B} 38$.

(C) 1988 American Mathematical Society $0002-9939 / 88 \$ 1.00+\$ .25$ per page 
derivative $h_{3}$ is given by $h_{3}=\left(h_{1}\right)(g)$, where $g$ is the unique $\Sigma$-measurable function satisfying $g \circ T_{1}=E\left(h_{2} \mid T_{1}^{-1}(\Sigma)\right)$. (Here, $E\left(\cdot \mid T_{1}^{-1}(\Sigma)\right)$ denotes the conditional expectation of - with respect to the sub- $\sigma$-algebra $T_{1}^{-1}(\Sigma)$.) $T_{3}$ and $h_{3}$ will satisfy conditions (i) and (ii) also. It is shown in [2] that $h \circ T \leq h$ a.e. is sufficient for the hyponormality of $C_{T}$. The main result of this paper is that if $h_{1} \circ T_{1} \leq h_{j}$ for $i, j=1,2$, , then for each of the positive integers $m, n$, and $p$ the operator $\left[C_{T_{1}}^{m} C_{T_{2}}^{n}\right]^{p}$ is hyponormal. As a consequence, we see that Harrington and Whitley's sufficient condition for hyponormality of a composition operator is actually sufficient for all powers to be hyponormal. In the last section of this paper, we give an example of a hyponormal composition operator whose square is not hyponormal.

We close this section with a useful observation. In [2, p. 130], Harrington and Whitley show that for every $g \in L^{2}$

$$
\langle(h \circ T) P g, g\rangle \geq\langle(h \circ T) g, g\rangle,
$$

where $T$ and $h$ are as above. Similar calculations may be used to prove the following lemma, whose proof we omit.

1.2 LEMMA. With $h, T$, and $P$ as above,

$$
\left.\left\langle\left(h^{n} \circ T\right) P g, g\right\rangle \leq\left(h^{n} \circ T\right) g, g\right\rangle, \quad n \in \mathbf{N}, g \in L^{2} .
$$

II. Main result and corollaries. In order to prove our main result (Theorem 1), it is necessary to state and prove several lemmas. They are stated so that each lemma depends on some subset of the previously stated ones. The proofs which are given contain the essential ideas, and may easily be adjusted to give the ones we omit.

2.1 LEMMA. If $h \circ T \leq h$ a.e. $m$, then for all $n \in \mathbf{N}, f \in L^{2}$ we have

$$
\left\langle h^{n} f, f\right\rangle \leq\left\langle\left(C_{T}^{n}\right)^{*} C_{T}^{n} f, f\right\rangle .
$$

ProOF. For $n=1$ the lemma is true by (1). Suppose (6) holds for $n=1,2, \ldots, k$ and all $f \in L^{2}$. Then

$$
\begin{aligned}
\left\langle\left(C_{T}^{k+1}\right)^{*} C_{T}^{k+1} f, f\right\rangle & =\left\langle\left(C_{T}^{k} C_{T}\right)^{*} C_{T}^{k} C_{T} f, f\right\rangle=\left\langle\left(C_{T}^{k}\right)^{*} C_{T}^{k}\left(C_{T} f\right),\left(C_{T} f\right)\right\rangle \\
& \geq\left\langle h^{k} C_{T} f, C_{T} f\right\rangle, \quad \text { for each } f \in L^{2},
\end{aligned}
$$

by the inductive hypothesis. But $\left\langle h^{k} C_{T} f, C_{T} f\right\rangle=\int h^{k}\left(|f|^{2} \circ T\right) d m$. By hypothesis, $h \geq h \circ T(\geq 0)$ a.e., so that $h^{k} \geq h^{k} \circ T$ a.e., and we have

$$
\left\langle h^{k} C_{T} f, C_{T} f\right\rangle \geq \int\left(h^{k} \circ T\right)\left(|f|^{2} \circ T\right) d m=\int\left(h^{k}|f|^{2}\right) h d m=\left\langle h^{k+1} f, f\right\rangle,
$$

so that (6) holds for $n=k+1$, and (2.1) follows by induction.

2.2 LEMMA. If $h \circ T \leq h$ a.e., then for all $n \in \mathrm{N}, f \in L^{2}$, we have

$$
\left\langle C_{T}^{n}\left(C_{T}^{n}\right)^{*} f, f\right\rangle \leq\left\langle(h \circ T)^{n} f, f\right\rangle \leq\left\langle h^{n} f, f\right\rangle .
$$

Proof. Again we will induct on $n$. For $n=1$ and $f \in L^{2}$,

$$
\left\langle C_{T} C_{T}^{*} f, f\right\rangle=\langle(h \circ T) P f, f\rangle \leq\langle(h \circ T) f, f\rangle,
$$

by Lemma 1.2. But

$$
\langle(h \circ T) f, f\rangle=\int(h \circ T)|f|^{2} d m \leq \int h|f|^{2} d m=\langle h f, f\rangle,
$$


since $0 \leq h \circ T \leq h$ a.e. Calculations similar to those used in the proof of Lemma 2.1 complete this proof by induction.

Now with $T_{1}, T_{2}$ as above, we set $A=C_{T_{1}}$ and $B=C_{T_{2}}$, so that the product $A B$ is the operator $C_{T_{3}}$.

2.3 LEMMA. With $A, B, h_{1}$, and $h_{2}$ as above, if

$$
\begin{aligned}
& \text { (a) } h_{2} \circ T_{2} \leq h_{1} \text { a.e., and } \\
& \text { (b) } h_{1} \circ T_{1} \leq h_{1} \text { a.e., }
\end{aligned}
$$

then for each $m, n \in \mathbf{N}$ and $f \in L^{2}$,

$$
\left\langle\left(A^{m} B^{n}\right)^{*}\left(A^{m} B^{n}\right) f, f\right\rangle \geq\left\langle h_{2}^{m+n} f, f\right\rangle .
$$

PrOOF. First we prove

2.3.1 Claim. With $T$ and $h$ as in $\S I$, for all $r, m \in \mathbf{N}$ and $f \in L^{2}$, we have

$$
\left\langle(h \circ T)^{r} C_{T}^{m} f, C_{T}^{m} f\right\rangle=\left\langle h^{r+m} f, f\right\rangle .
$$

Proof of claim. Fix $r$ and induct on $m$. For $m=1$ and $f \in L^{2}$,

$$
\begin{aligned}
\left\langle(h \circ T)^{r} C_{T} f, C_{T} f\right\rangle & =\int(h \circ T)^{r}\left(|f|^{2} \circ T\right) d m \\
& =\int\left(h^{r}|f|^{2}\right) h d m=\left\langle h^{r+1} f, f\right\rangle .
\end{aligned}
$$

Suppose (12) holds for $m=k$ and all $f \in L^{2}$. Then

$$
\begin{aligned}
\left\langle(h \circ T)^{r} C_{T}^{k+1} f, C_{T}^{k+1} f\right\rangle & =\left\langle(h \circ T)^{r+k} C_{T} f, C_{T} f\right\rangle \\
& =\int\left(h^{r+k} \circ T\right)\left(|f|^{2} \circ T\right) d m=\left\langle(h)^{r+k+1} f, f\right\rangle,
\end{aligned}
$$

and (12) holds for $m=k+1$. The claim is proved by induction.

To finish the proof of Lemma 2.3, observe that

$$
\begin{aligned}
\left\langle\left(A^{m} B^{n}\right)^{*}\left(A^{m} B^{n}\right) f, f\right\rangle & =\left\langle\left[\left(A^{m}\right)^{*}\left(A^{m}\right)\right] B^{n} f, B^{n} f\right\rangle \\
& \geq\left\langle h_{1}^{m} B^{n} f, f\right\rangle \quad \text { (by Lemma 2.1) } \\
& \geq\left\langle\left(h_{2} \circ T_{2}\right)^{m} B^{n} f, B^{n} f\right\rangle \quad \text { (by hypothesis) } \\
& =\left\langle h_{2}^{m+n} f, f\right\rangle \quad \text { (by Claim 2.3.1). }
\end{aligned}
$$

2.4 LEMMA. With $A, B, h_{1}$, and $h_{2}$ as above, if

$$
h_{i} \circ T_{i} \leq h_{j}, \quad i, j=1,2,
$$

then for each $m, n \in \mathbf{N}$ and $f \in L^{2}$,

$$
\left\langle\left(A^{m} B^{n}\right)\left(A^{m} B^{n}\right)^{*} f, f\right\rangle \leq\left\langle h_{2}^{m+n} f, f\right\rangle .
$$

ProOF. First we prove

2.4.1 Claim. If $h \circ T \leq h$ a.e., then for all $r, m \in \mathrm{N}, f \in L^{2}$,

$$
\left\langle h^{r}\left(C_{T}^{m}\right)^{*} f,\left(C_{T}^{m}\right)^{*} f\right\rangle \leq\left\langle(h \circ T)^{r+m} f, f\right\rangle .
$$

Proof of claim. Fix $r$ and induct on $m$. For $m=1$ and $f \in L^{2}$,

$$
\begin{aligned}
\left\langle h^{r} C_{T}^{*} f, C_{T}^{*}, f\right\rangle & =\left\langle\left(h^{r} \circ T\right) C_{T} C_{T}^{*} f, f\right\rangle \\
& =\left\langle\left(h^{r+1} \circ T\right) P f, f\right\rangle \quad(\text { by Lemma 1.1) } \\
& \left.\leq\left(h^{r+1} \circ T\right) f, f\right\rangle \quad(\text { by Lemma 1.2). }
\end{aligned}
$$


Completing the induction step is similar and is left to the reader. To finish the proof of Lemma 2.4, note that

$$
\begin{aligned}
\left\langle\left(A^{m} B^{n}\right)\left(A^{m} B^{n}\right)^{*} f, f\right\rangle & =\left\langle\left[B^{n}\left(B^{n}\right)^{*}\right]\left(A^{m}\right)^{*} f,\left(A^{m}\right)^{*} f\right\rangle \\
& \leq\left\langle\left(h_{2} \circ T_{2}\right)^{n}\left(A^{m}\right)^{*} f,\left(A^{m}\right)^{*} f\right\rangle \quad \text { (by Lemma 2.2) } \\
& \leq\left\langle h_{1}^{n}\left(A^{m}\right)^{*} f,\left(A^{m}\right)^{*} f\right\rangle \quad \text { (by hypothesis) } \\
& \leq\left\langle\left(h_{1} \circ T_{1}\right)^{n+m} f, f\right\rangle \quad \text { (by Claim 2.4.1) } \\
& \leq\left\langle h_{2}^{n+m} f, f\right\rangle \quad \text { (by hypothesis). } \quad
\end{aligned}
$$

Finally, similar techniques and the above lemmas may be used to prove the following pair of inequalities, which we collect as Lemma 2.5 and state without proof.

2.5 LEMMA. If (13) holds, then for all $m, n, p \in \mathbf{N}$ and $f \in L^{2}$, we have

$$
\left\langle\left[\left(A^{m} B^{n}\right)^{p}\right]^{*}\left(A^{m} B^{n}\right)^{p} f, f\right\rangle \geq\left\langle h_{2}^{(m+n) p} f, f\right\rangle
$$

and

$$
\left.\left\langle\left[A^{m} B^{n}\right]^{p}\left(A^{m} B^{n}\right)^{p}\right]^{*} f, f\right\rangle \leq\left\langle h_{2}^{(m+n) p} f, f\right\rangle .
$$

REMARK. $h_{2} \circ T_{2} \leq h_{2}$ is not necessary in the proof of (16). Now we may easily prove our main result:

THEOREM 1. If (13) holds, $\left(A^{m} B^{n}\right)^{p}$ is hyponormal for all $m, n, p \in \mathbf{N}$.

PROOF. By first applying (16) and then applying (17) we have, for $m, n, p \in \mathbf{N}$ and $f \in L^{2}$,

$$
\left\langle\left[\left(A^{m} B^{n}\right)^{p}\right]^{*}\left(A^{m} B^{n}\right)^{p} f, f\right\rangle \geq\left\langle h_{2}^{(m+n) p} f, f\right\rangle \geq\left\langle\left[A^{m} B^{n}\right]^{p}\left[\left(A^{m} B^{n}\right)^{p}\right]^{*} f, f\right\rangle,
$$

so that $\left\|\left(A^{m} B^{n}\right)^{p} f\right\| \geq\left\|\left[\left(A^{m} B^{n}\right)^{p}\right]^{*} f\right\|$.

The following corollaries are immediate.

COROLlaRY 1. With $A, B, h_{1}$, and $h_{2}$ as above, if (13) holds, then for each $m, n \in \mathbf{N}, A^{m} B^{n}$ is hyponormal.

REMARK. This may be proved directly using Lemmas 2.3 and 2.4.

COROLlaRY 2. If (13) holds, then $(A B)^{p}$ is hyponormal for each $p \in \mathbf{N}$.

REMARK. Observe that (13) implies a priori that both $A$ and $B$ are hyponormal. Actually, the conclusion of Corollary 2 is true under the weaker hypothesis that $h_{i} \circ T_{i} \leq h_{j}, i \neq j, i, j=1,2$. These hypotheses may be used to prove the inequalities

$$
\left\langle\left[(A B)^{p}\right]^{*}(A B)^{p} f, f\right\rangle \geq\left\langle h_{2}^{2 p} f, f\right\rangle
$$

and

$$
\left\langle(A B)^{p}\left[(A B)^{p}\right]^{*} f, f\right\rangle \leq\left\langle h_{2}^{2 p} f, f\right\rangle,
$$

from which Corollary 2 follows immediately.

COROLlaRY 3. With $T, h$, and $m$ as in $\S \mathrm{I}$, if $h \circ T \leq h$ a.e. $m$, then $C_{T}^{n}$ is hyponormal for each $n \in \mathbf{N}$.

REMARK. Corollary 3 also follows directly from Lemmas 2.1 and 2.2. 
III. An example. Halmos notes in [1] that it is not easy to find examples of hyponormal operators whose squares are not hyponormal. The simplest such example is due to Ito and Wong [3]; namely, that $U^{*}+2 U$, where $U$ is the unilateral shift on $l^{2}(\mathbf{N})$, is hyponormal but its square is not. For a hyponormal composition operator whose square is not hyponormal, we have the following

EXAMPLE. Let $X=[0, \infty)$ be equipped with Lebesgue measure on the Borel sets. Set

$$
\begin{aligned}
T x= & x \chi_{[0,1)}+(10 x / 3-10 / 3) \chi_{(1,13 / 10]}+(5 x / 2-9 / 4) \chi_{(13 / 10,3 / 2]} \\
& +(3 x / 10-9 / 20) \chi_{(3 / 2,29 / 6]}+(x / 100+871 / 600) \chi_{(29 / 6, \infty)}
\end{aligned}
$$

Then

$$
h=d m \circ T^{-1} / d m=139 / 30 \chi_{[0,1)}+2 / 5 \chi_{(1,3 / 2]}+100 \chi_{(3 / 2, \infty)} .
$$

$\left(\chi_{[a, b)}\right.$ stands for the characteristic function of $[a, b)$.) Using Lemma 15 of [2], it can be shown that $C_{T}$ is hyponormal. If one considers $g(x)=\chi_{(1,13 / 10]}$, then a direct but tedious computation shows that $\left\langle C_{T}^{2 *} C_{T}^{2} g, g\right\rangle<\left\langle C_{T}^{2} C_{T}^{2 *} g, g\right\rangle$ so that $C_{T}^{2}$ is not hyponormal.

\section{BIBLIOGRAPHY}

1. P. R. Halmos, A Hilbert space problem book, Van Nostrand, Princeton, N. J., 1976.

2. D. J. Harrington and R. Whitley, Seminormal composition operators, J. Operator Theory 11 (1984), 125-135.

3. T. Ito and T. K. Wong, Subnormality and quasinormality of Toeplitz operators, Proc. Amer. Math. Soc. 34 (1972), 157-164.

4. A. Lambert, Hyponormal composition operators, Bull. London Math. Soc. 18 (1986), 395-400.

5. E. Nordgren, Composition operators in Hilbert space, Hilbert Space Operators (Proc. 1977), Lecture Notes in Math., vol. 693, Springer-Verlag, Berlin, 1978, pp. 37-63.

Department of Mathematical Sciences, Memphis State University, Memphis, TENNESSEE 38152 